\title{
THE THREE-DIMENSIONAL STRESS INTENSITY FACTOR DUE TO THE MOTION OF A LOAD ON THE FACES OF A CRACK*
}

\author{
BY \\ JEAN - CLAUDE RAMIREZ \\ Brown University
}

\begin{abstract}
The dynamic stress intensity factor history for a half plane crack in an otherwise unbounded elastic body, with the crack faces subjected to a traction distribution consisting of a pair of point loads that move in a direction perpendicular to the crack edge, is considered. The exact expression for the mode I stress intensity factor as a function of time for any point along the crack edge is obtained by extending a procedure recently introduced by Freund [1]. The method of solution is based on integral transform methods and the theory of analytic functions of a complex variable. Some features of the solution are discussed and graphical results for various point load speeds are presented.
\end{abstract}

1. Introduction. A general procedure has been introduced by Freund [1] for determining the stress intensity factor histories for a class of three-dimensional elastodynamic crack problems. As an illustration of the procedure, Freund studied a half plane crack in an otherwise unbounded elastic solid, with the crack faces subjected to a pair of line loads that are suddenly applied along a line perpendicular to the crack front. Because the approach is novel, its range of applicability has not yet been established. Here, the extension of the procedure to situations with moving loads on the crack faces is considered. This distribution consists of a pair of point loads that suddenly begins to act at the edge of the crack and moves at a constant velocity along the crack faces in a direction perpendicular to the crack edge. The corresponding two-dimensional problem was studied by Ang [2]. A three-dimensional problem that is related to the one in this paper is that of a point load traveling on the surface of an elastic half space. This problem was analyzed by Gakenheimer and Miklowitz [3], who considered all point load speeds, i.e., subsonic, transonic, and supersonic. For the purposes of this paper, attention is restricted to subsonic point load speeds; that is, the speed is less than the characteristic Rayleigh wave speed of the material. The analysis for higher speeds offers no added mathematical difficulty.

\footnotetext{
* Received June 4, 1986.
} 
In Sec. 2, the general formulation of the boundary value problem is presented for the three-dimensional crack face tractions resulting in mode I deformation. Section 3 describes the general approach to solving the problem by means of transform methods. In Sec. 4, the formal solution to the particular traction distribution is obtained by means of the Wiener-Hopf decomposition method. In Sec. 5 the dynamic stress intensity factor history is extracted by making use of the asymptotic properties of transforms, the Cagniard-deHoop method, and the convolution theorem for transforms. A discussion of the results is given in Sec. 6 .

2. General formulation. In vector notation the Navier equation governing the displacement vector $\mathbf{u}$ for an isotropic elastic solid is written as

$$
\ddot{\mathbf{u}}=c_{1}^{2} \nabla(\nabla \cdot \mathbf{u})-c_{s}^{2} \nabla \times(\nabla \times \mathbf{u}),
$$

where $c_{1}$ and $c_{s}$ are the dilatational and shear wave speeds, respectively. In terms of the Lamé constants $\lambda$ and $\mu$ and the mass density $\rho$, the wave speeds are given by

$$
c_{1}^{2}=(\lambda+2 \mu) / \rho, \quad c_{s}^{2}=\mu / \rho .
$$

It is also useful to introduce the dilatational and shear slownesses $a$ and $b$, where $a=1 / c_{1}$ and $b=1 / c_{s}$. Furthermore, the Rayleigh wave speed of the elastic material is denoted by $c_{r}$ and its corresponding slowness by $r$.

A standard approach when solving (2.1) is to introduce the displacement potentials $\varphi$ and $\psi$ through the Helmholtz decomposition of the displacement vector, i.e.,

$$
\mathbf{u}=\nabla \varphi+\nabla \times \psi, \quad \nabla \cdot \psi=0 .
$$

The scalar potential $\varphi$ is called the dilatational potential and the vector potential $\psi$ is the shear potential. The divergence free requirement on the shear potential is necessary in order to make the decomposition unique. The advantage of this decomposition is that the potentials $\varphi$ and $\psi$ satisfy the uncoupled wave equations

$$
\ddot{\varphi}=c_{1}^{2} \nabla^{2} \varphi, \quad \ddot{\psi}=c_{s}^{2} \nabla^{2} \psi .
$$

The linear differential equations (2.4) have the added advantage of lending themselves to standard integral transform methods. The two potentials are coupled through the boundary conditions that characterize the problem to be described.

Consider the elastic body containing a half plane crack depicted in Fig. 1. A right-handed rectangular coordinate system is introduced such that the $z$-axis coincides with the crack front, and the half plane crack occupies the region $y=0, x<0$. Attention is restricted to applied tractions of the form $T_{y}=\mp \sigma_{-}(x, z, t)$ on $y= \pm 0$, where $\sigma_{-}>0$ corresponds to a tensile traction. All other components of the imposed traction are zero. The function $\sigma_{-}$is prescribed for $x<0$ and is extended so that $\sigma_{-} \equiv 0$ in the half range $x>0$. The minus subscript is used to denote a function that is nonvanishing in the range $x<0$. Likewise, the plus subscript will be used to label functions that are nonzero in the half range $x>0$ but are identically zero for $x<0$. This notation is useful in problems like this one, where the transforms of these "half functions" turn out to be analytic functions of the transform parameter in lower (minus) and upper (plus) half planes. 


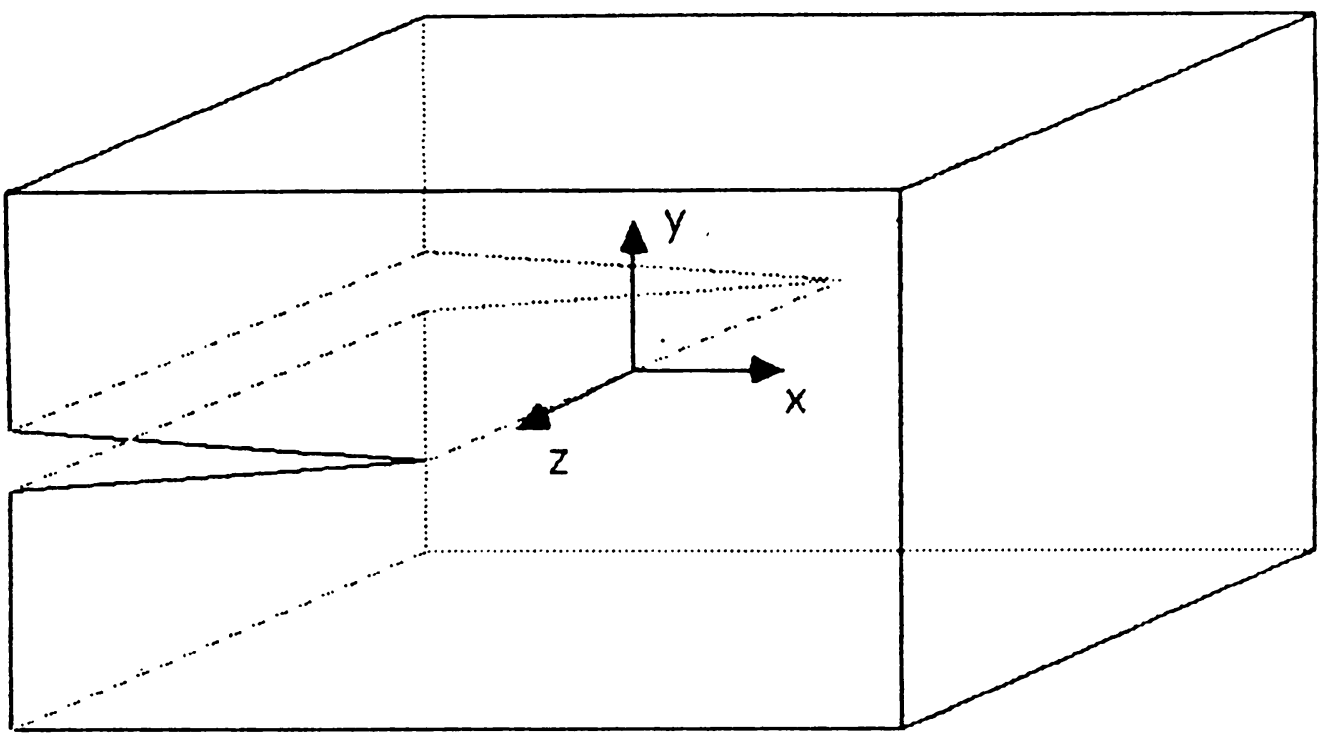

FIG. 1. Geometrical configuration of the elastic solid.

Due to the symmetry of the geometry and the applied traction, the displacement fields satisfy the following conditions:

$$
\begin{aligned}
& u_{x}(x,-y, z, t)=u_{x}(x, y, z, t), \\
& u_{y}(x,-y, z, t)=-u_{y}(x, y, z, t), \\
& u_{z}(x,-y, z, t)=u_{z}(x, y, z, t) .
\end{aligned}
$$

Thus attention can be restricted to the upper half space $y \geqslant 0$. Furthermore, properties (2.5) imply the $\sigma_{x y}(x, 0, z, t)$ and $\sigma_{y z}(x, 0, z, t)$ vanish for all $z$ and $t$ and that $u_{y}(x, 0, z, t)$ $=u_{-}(x, z, t)$ where $u_{-}$represents the unknown $y$ component of the displacement on the crack faces for $x<0$ and $u_{-} \equiv 0$ in the half range $x>0$. Hence, the complete set of boundary conditions to be satisfied by the stress field is

$$
\begin{aligned}
& \sigma_{y y}(x, 0, z, t)=\sigma_{-}(x, z, t)+\sigma_{+}(x, z, t), \\
& \sigma_{x y}(x, 0, z, t)=0, \\
& \sigma_{y z}(x, 0, z, t)=0, \\
& u_{y}(x, 0, z, t)=u_{-}(x, z, t),
\end{aligned}
$$

for $-\infty<x, z<\infty$ and $0 \leqslant t$. The function $\sigma_{+}$represents the unknown normal component of stress $\sigma_{y y}$ on $x>0$, and $\sigma_{+} \equiv 0$ for $x<0$.

The initial conditions are that the material is stress-free and at rest for $t \leqslant 0$. These are expressed in terms of the displacement potentials by

$$
\varphi(x, y, z, 0)=\partial_{t} \varphi(x, y, z, 0)=0, \quad \psi(x, y, z, 0)=\partial_{t} \psi(x, y, z, 0)=\mathbf{0}
$$

for $y>0$. Likewise, the boundary conditions (2.6) can be replaced by their corresponding representations in terms of $\varphi$ and $\psi$. 
3. Transforms. In order to solve the problem described by the partial differential equations (2.4), the initial conditions (2.7), and the boundary conditions (2.6), use is made of Laplace and Fourier transforms. First, the one-sided Laplace transform is applied to the differential equations and the boundary conditions, and the initial conditions are imposed. This Laplace transform in time has the transform parameter $s$ and is denoted by a superposed hat. For a function $\varphi(x, y, z, t)$ this transform is given by

$$
\hat{\varphi}(x, y, z, s)=\int_{0}^{\infty} \varphi(x, y, z, t) e^{-s t} d t .
$$

For the time being, $s$ is taken to be real and large enough to be on the right of the abscissa of convergence of the integral. The dependence on $z$ and $x$ is then suppressed by applying a pair of two-sided Fourier transforms. These transforms have the transform parameters $i \zeta s$ and $i \xi s$, respectively. They are denoted, respectively, by the uppercase symbol for the function with a superposed hat and the uppercase symbol itself, to wit,

$$
\begin{aligned}
& \hat{\Phi}(x, y, \zeta, s)=\frac{1}{\sqrt{2 \pi}} \int_{-\infty}^{\infty} \hat{\varphi}(x, y, z, s) e^{i s \zeta z} d z, \\
& \Phi(\xi, y, \zeta, s)=\frac{1}{\sqrt{2 \pi}} \int_{-\infty}^{\infty} \hat{\Phi}(x, y, \zeta, s) e^{i s \xi x} d x .
\end{aligned}
$$

The introduction of the $s$ parameter into the kernel of the transforms (3.2) is for algebraic convenience; it takes care of a change of variables that would otherwise be needed at a later point in the analysis.

Due to the wave propagation character of the solution, the strip of analyticity of the Fourier transforms can be anticipated. Suppose that the applied tractions are such that $\sigma_{-}$ vanishes for $|z|>z_{0}$. Then, at any given time $t$, the region of causality is confined to $|z|<z_{0}+t c_{1}$. Thus by considering the elementary wave field $\varphi=H\left[t+\left(z+z_{0}\right) a\right]+$ $H\left[t-\left(z-z_{0}\right) a\right]$, where $H(\cdot)$ is Heaviside's unit function, and applying to it the Laplace transform, one obtains

$$
\hat{\Phi}=O\left(e^{-s\left(|z|-z_{0}\right) a}\right) \quad \text { as }|z| \uparrow \infty .
$$

This, in turn, implies that the Fourier transform $\hat{\Phi}$ converges for the strip $|\operatorname{Im}(\zeta)|<a$. Thus $\hat{\Phi}$ defines an analytic function of $\zeta$ in the strip of convergence. Consequently, one can analytically continue $\hat{\Phi}$ to values of $\zeta$ that are not contained in the strip of convergence. At this point in the analysis it is convenient to restrict $\zeta$ to this strip. The identity property of analytic functions [4] allows one to restrict $\zeta$ to the portion of the imaginary axis in the interval $-a<\operatorname{Im}(\zeta)<a, \operatorname{Re}(\zeta)=0$. The idea is to perform the Wiener-Hopf factorization in the $\xi$ plane only, keeping $\zeta$ confined to the strip of analyticity of $\hat{\Phi}$. At a later point in the analysis it will be essential to analytically extend functions of $\zeta$ away from the interval on the imaginary axis and outside the strip.

The domain of convergence of the Fourier transform in $x$ can also be anticipated. Suppose that the applied tractions are such that along the crack faces $\sigma_{-}$is nonzero for indefinitely large values of $x$ in the negative direction. Then, in this region the integral $\hat{\Phi}$ will converge provided that $\operatorname{Im}(\xi)<0$. On the other hand, by definition the applied tractions do not extend along the positive $x$ direction, and thus the region of causality 
does not extend beyond a certain cylindrical wave front ahead of the crack front. To be precise, for $x>0$, the front is centered at the $y$-axis and, at any given time $t$, has a radius $z_{0}+\left(x^{2}+z^{2}\right)^{1 / 2}$. Thus, by considering the elementary wave field $\varphi=H\left(t-\left(z_{0}\right.\right.$ $\left.+\sqrt{x^{2}+z^{2}}\right) a$ ) and applying to it the transforms (3.1) and (3.2) it is found that the final integral converges if $\{\operatorname{Im}(\xi)\}^{2}-\xi^{2}<a^{2}$. Therefore the Fourier integral $\Phi$ defines an analytic function in the strip $-\sqrt{\zeta^{2}+a^{2}}<\operatorname{Im}(\xi)<0$ in the $\xi$-plane, with $\zeta$ restricted to $-a<\operatorname{Im}(\zeta)<a, \operatorname{Re}(\zeta)=0$.

The class of problems which is accessible by the solution procedure outlined in the Introduction is one in which $\sigma_{-}(x, z, t)$ is restricted to having a triple transform which has the separable form

$$
\frac{1}{2 \pi} \int_{-\infty}^{\infty} \int_{-\infty}^{\infty} \hat{\sigma}_{-}(x, z, s) e^{i s(\xi x+\zeta z)} d z d x=\frac{1}{s^{m}} \Sigma_{-}(\xi, \zeta),
$$

where $m$ is a real number and $\Sigma_{-}(\xi, \zeta)$ does not depend on $s$. The reason for this will be more apparent in Sec. 5. The requirement (3.4) makes it possible to perform the final inversion of the dynamic stress intensity factor by means of the convolution formula for Laplace transforms.

The application of the transforms (3.1) and (3.2) to the partial differential equations (2.4) reduces them to ordinary differential equations,

$$
\frac{\partial^{2} \Phi}{\partial y^{2}}-s^{2} \alpha^{2} \Phi=0 \quad \text { and } \quad \frac{\partial^{2} \Psi}{\partial y^{2}}-s^{2} \beta^{2} \Psi=\mathbf{0},
$$

where

$$
\alpha=\alpha(\zeta, \xi)=\sqrt{\zeta^{2}+\xi^{2}+a^{2}}, \quad \beta=\beta(\zeta, \xi)=\sqrt{\zeta^{2}+\xi^{2}+b^{2}},
$$

and $a=1 / c_{1}, b=1 / c_{s}$ are the dilatational and shear slownesses introduced after (2.2). The complex $\xi$-plane is cut along $\sqrt{a^{2}+\zeta^{2}}<|\operatorname{Im}(\xi)|<\infty, \operatorname{Re}(\xi)=0$ and $\sqrt{b^{2}+\zeta^{2}}<$ $|\operatorname{Im}(\xi)|<\infty, \operatorname{Re}(\xi)=0$ so that $\operatorname{Re}(\alpha) \geqslant 0$ and $\operatorname{Re}(\beta) \geqslant 0$ in the cut plane for each admissible value of $\zeta$. With the $\xi$-plane cut in this fashion, equations of the type (3.5) have the solutions, bounded as $y \rightarrow \infty$ to preclude waves coming in from remote regions,

$$
\Phi=\frac{A(\xi, \zeta)}{s^{m+2}} e^{-s \alpha y}, \quad \Psi=\frac{\Theta(\xi, \zeta)}{s^{m+2}} e^{-s \beta y},
$$

where $\Theta=\{B(\xi, \zeta), C(\xi, \zeta), D(\xi, \zeta)\}$.

Transforming the condition $\nabla \cdot \psi=0$ yields

$$
\xi B-i \beta C+\zeta D=0 .
$$

It is also necessary to transform the boundary conditions (2.6), which will yield four more equations. They are

$$
\begin{aligned}
\left(b^{2}+2 \xi^{2}+2 \zeta^{2}\right) A+2 i \zeta \beta B-2 i \xi \beta D & =\mu^{-1}\left(\Sigma_{-}+\Sigma_{+}\right), \\
2 i \zeta \alpha A-\xi \zeta B-i \zeta \beta C+\left(\beta^{2}+\xi^{2}\right) D & =0 \\
2 i \zeta \alpha A-\left(\beta^{2}+\zeta^{2}\right) B+i \xi \beta C+\xi \zeta D & =0 \\
-\alpha A-i \zeta B+i \xi D & =U_{-} .
\end{aligned}
$$


In reducing the equations to this form, use is made of the fact that $\lambda c_{l}^{-2}=\mu\left(b^{2}-2 a^{2}\right)$. Furthermore, we have defined $U_{-}$and $\Sigma_{+}$as

$$
\begin{aligned}
U_{-}(\xi, \zeta) & =\frac{s^{m+1}}{2 \pi} \int_{-\infty}^{\infty} \int_{-\infty}^{\infty} \hat{u}_{-}(x, z, s) e^{i s(\xi x+\zeta z)} d z d x, \\
\Sigma_{+}(\xi, \zeta) & =\frac{s^{m}}{2 \pi} \int_{-\infty}^{\infty} \int_{-\infty}^{\infty} \hat{\sigma}_{-}(x, z, s) e^{i s(\xi x+\zeta z)} d z d x .
\end{aligned}
$$

The parameter $s$ is absent, by construction, from the five equations (3.8) and (3.9). There are six unknown parameters, four constants of integration $A, B, C, D$, and two sectionally analytic funtions $U_{-}$and $\Sigma_{+}$; but only five equations to relate them. One can solve for the four constants of integration in terms of the two unknown sectionally analytic functions. The result is

$$
\begin{aligned}
& A(\xi, \zeta)=\alpha^{-1}\left(1-2 \beta^{2} b^{-2}\right) U_{-}(\xi, \zeta), \\
& B(\xi, \zeta)=-2 i \zeta b^{-2} U_{-}(\xi, \zeta), \\
& C(\xi, \zeta)=0 \\
& D(\xi, \zeta)=2 i \xi b^{-2} U_{-}(\xi, \zeta) .
\end{aligned}
$$

Substituting for these functions into the first equation of (3.9), we obtain one equation relating the two unknown functions,

$$
-\frac{\mu}{b^{2} \alpha} R(\xi, \zeta) U_{-}(\xi, \zeta)=\Sigma_{-}(\xi, \zeta)+\Sigma_{+}(\xi, \zeta)
$$

where

$$
R(\xi, \zeta)=\left[b^{2}+2\left(\xi^{2}+\zeta^{2}\right)\right]^{2}-4\left(\xi^{2}+\zeta^{2}\right) \alpha(\xi, \zeta) \beta(\xi, \zeta) .
$$

This is the modified Rayleigh wave function; that is, it corresponds to the standard Rayleigh wave function, $R(z)$, when $\zeta=0$. It is a well-established fact [5] that the standard Rayleigh wave function, in a properly cut $z$-plane, has only two zeros, $R( \pm i r)$ $=0$, whre $r=1 / c_{r}$. Thus, the modified Rayleigh wave function in the properly cut complex $\xi$-plane has only the two zeros $\xi= \pm i \bar{r}$, where $\bar{r}=\sqrt{\zeta^{2}+r^{2}}$.

Equation (3.12) is a standard Wiener-Hopf equation, and the essence of the Wiener-Hopf method is to solve for the two unknown sectionally analytic funtions $U_{-}$and $\Sigma_{+}$from a single equation (3.12). According to Noble [6], the approach to be followed in Sec. 4 is actually due to Jones. Since $\Sigma_{+}$is analytic in the half plane $\operatorname{Im}(\xi)>-\sqrt{\zeta^{2}+a^{2}}$, and $U_{-}$is analytic in the half plane $\operatorname{Im}(\xi)<0,(3.12)$ holds in the strip $-i \sqrt{\zeta^{2}+a^{2}}<\xi<0$. Furthermore, $\zeta$ is restricted to the interval $-a<\operatorname{Im}(\zeta)<a, \operatorname{Re}(\zeta)=0$, so for a fixed value of $\zeta$ (3.12) can be solved by factorization in the $\xi$-plane alone.

4. Formal solution. At this point it is necessary to introduce the particular applied traction distribution. The present work is concerned with a pair of point loads that suddenly begins to act at the edge of the crack $x=y=z=0$ and moves in the negative $x$ direction, i.e., perpendicular to the crack edge, at a contant velocity $v$. Thus, we assume that

$$
\sigma_{-}(x, z, t)=-P \delta(z) \delta(x+v t), \quad 0<v<c_{r},
$$

where $\delta(\cdot)$ is Dirac's delta function. The amplitude $P$ has physical dimensions of force and $P>0$ corresponds to a traction that tends to separate the crack faces. 
Figures 2,3, and 4 indicate the leading wave fronts that result from the application of the tractions (4.1). These wave fronts have been obtained by extrapolation from experience with two-dimensional problems in hyperbolic partial differential equations rather than by solving for the displacement potentials. The wave fronts include spherical dilatational and shear wave fronts centered at the origin of coordinates (Fig. 2). There are also two sets of head waves that form cones with vertices where the spherical dilatational front meets the $z$-axis, and extend to circles of tangency with the spherical shear wave front. The traces of these cones which extend to the crack faces, as well as ahead of the crack edge, are shown in Fig. 2. Furthermore, there are conical head waves that intersect the dilatational wave fronts on the surfaces $y= \pm 0$ for $x<0$ and extend to circles of tangency with the spherical shear wave fronts (Figs. 3 and 4). The head waves arise since the dilatational waves alone cannot satisfy the traction free boundary conditions that exist at the crack faces.

Transforming (4.1) with (3.1) and (3.2), it is found that $m=1$ and that

$$
\Sigma_{-}(\xi, \zeta)=\frac{i P}{2 \pi v} \frac{1}{(\zeta-i c)},
$$

where $c=1 / v$ is the point load slowness. Substituting for $\Sigma_{-}(\xi, \zeta)$ in (3.12), one obtains the Wiener-Hopf equation that corresponds to the problem with traction loading (4.1),

$$
-\frac{\mu}{b^{2} \alpha} R(\xi, \zeta) U_{-}(\xi, \zeta)=\frac{i P}{2 \pi v} \frac{1}{(\zeta-i c)}+\Sigma_{+}(\xi, \zeta) .
$$

Only some of the steps involved in the factorization of (4.3) in the $\xi$-plane will be shown below. To begin, let $\bar{a}^{2}=\zeta^{2}+a^{2}$ and note that $\bar{a}$ is some constant in the interval $(0, a)$ since $\zeta$ is being held fixed in the interval $(-i a, i a)$. The function $\alpha$ can be factorized as

$$
\alpha(\xi ; \zeta)=\left(\xi^{2}+\bar{a}^{2}\right)^{1 / 2}=\left[(\xi+i \bar{a})^{1 / 2}\right]_{+}\left[(\xi-i \bar{a})^{1 / 2}\right]_{-} .
$$

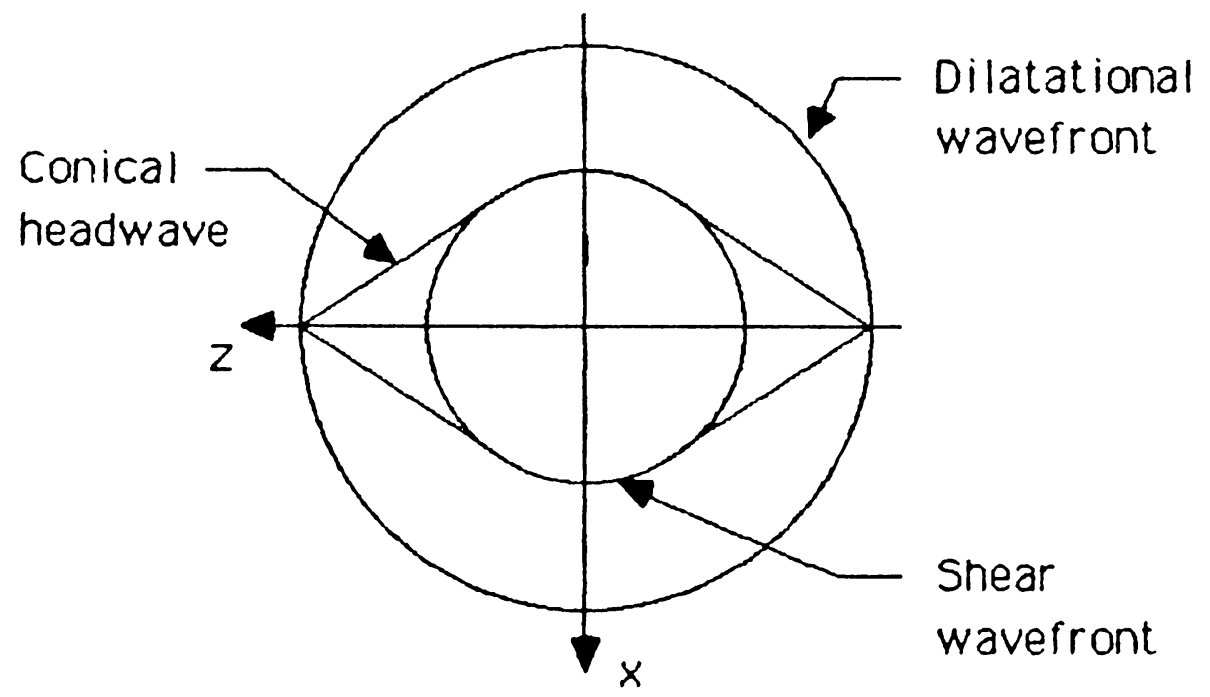

FIG. 2. Traces in the $x-z$ plane of the wave fronts resulting from the application of the tractions (4.1). 


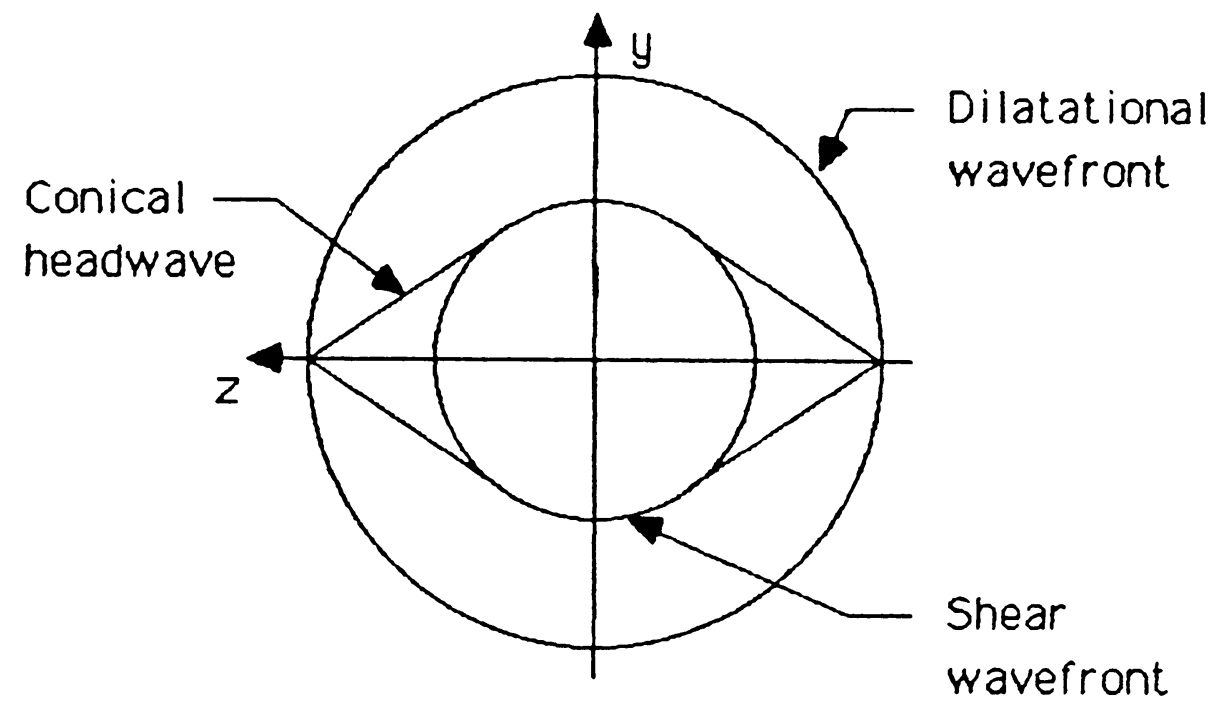

FIG. 3. Traces in the $y-z$ plane of the wave fronts resulting from the application of the tractions (4.1).

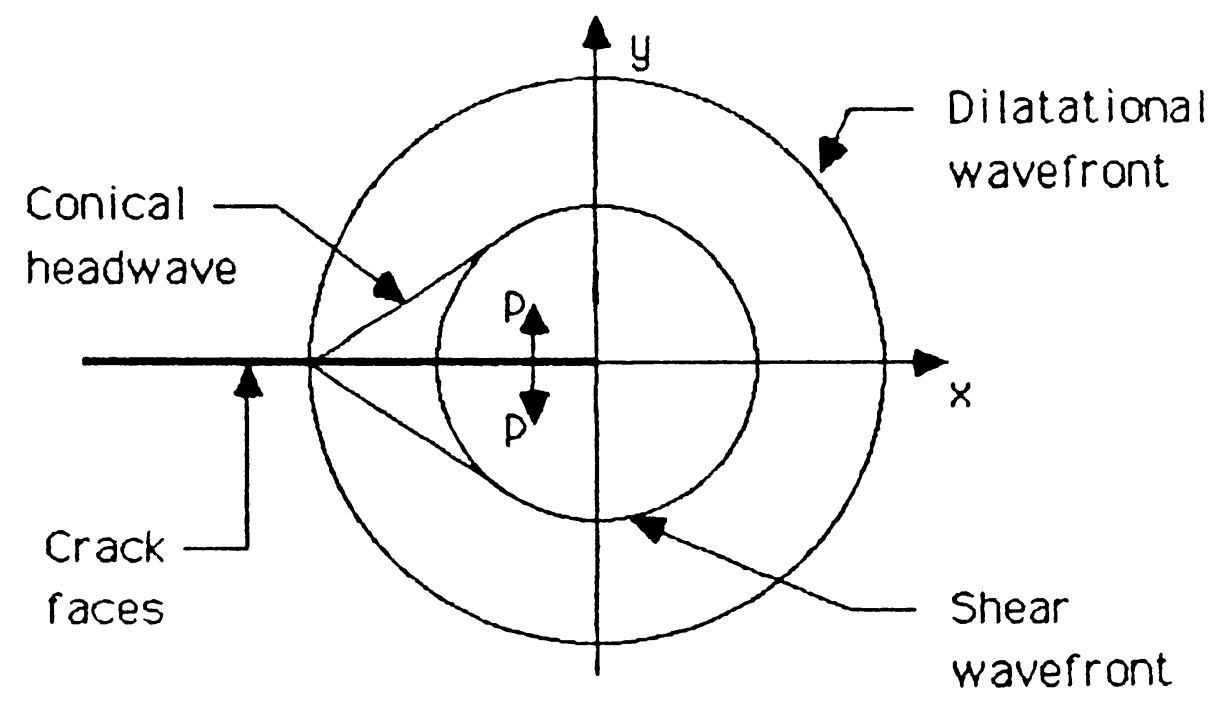

FIG. 4. Traces in the $x-y$ plane of the wave fronts resulting from the application of the tractions (4.1).

The semicolon is used to emphasize the fact that $\zeta$ is being held fixed and its influence in the factorization procedure is only parametric in nature. The plus (minus) subscript is used to denote functions that are analytic in the upper (lower) half plane $\operatorname{Im}(\xi)>-\bar{a}(<0)$.

In order to make further progress one must factorize the Rayleigh wave function $R(\xi ; \zeta)$. For this purpose it is most convenient to express $R(\xi ; \zeta)$ in terms of a function with neither zeros nor poles and whose limiting value as $|\xi| \uparrow \infty$ is unity ([6], pp. 13-15). Such a function is easily constructed from the asymptotic properties of the Rayleigh wave 
function and knowledge of its roots. Specifically, one must make use of the results that

$$
R(\xi ; \zeta)=2 \xi^{2}\left(b^{2}-a^{2}\right)+O(1) \quad \text { as }|\xi| \uparrow \infty
$$

and $R(\xi ; \zeta)$ has two symmetric roots $\xi= \pm i \bar{r}$, where $\bar{r}=\sqrt{\zeta^{2}+r^{2}}$, as discussed at the end of Sec. 3. The function needed is easily seen to be

$$
S(\xi ; \zeta)=\frac{R(\xi ; \zeta)}{\kappa\left(\bar{r}^{2}+\xi^{2}\right)},
$$

where $\kappa=2\left(b^{2}-a^{2}\right)$. The function $S$ has the required properties of being nonvanishing in the finite $\xi$-plane and being asymptotic to unity for large values of $\xi$. It may be shown that $S(\xi ; \zeta)$ can be factorized as $S_{+}(\xi ; \zeta) S_{-}(\xi ; \zeta)$, where

$$
S_{ \pm}(\xi, \zeta)=\exp \left\{\frac{1}{\pi} \int_{a}^{b} \tan ^{-1}\left(\frac{4 \eta^{2} \sqrt{\left(b^{2}-\eta^{2}\right)\left(\eta^{2}-a^{2}\right)}}{\left(b^{2}-2 \eta^{2}\right)^{2}}\right) \frac{\eta d \eta}{\sqrt{\zeta^{2}+\eta^{2}}\left(\sqrt{\zeta^{2}+\eta^{2}} \pm \xi\right)}\right\} \text {. }
$$

The functions $S_{+}(\xi ; \zeta)$ and $S_{-}(\xi ; \zeta)$ are analytic and nonzero in the half planes $\operatorname{Im}(\xi)>-\bar{a}$ and $\operatorname{Im}(\xi)<\bar{a}$.

After some straightforward manipulations, (4.3) can be rewritten as

$$
-\frac{\mu \kappa U_{-}(\xi ; \zeta)}{b^{2} G_{-}(\xi ; \zeta)}=\left[\frac{i P}{2 \pi v(\xi-i c)}+\Sigma_{+}(\xi ; \zeta)\right] G_{+}(\xi ; \zeta)
$$

where

$$
G_{ \pm}(\xi ; \zeta)=\frac{(\xi \pm i \bar{a})^{1 / 2}}{(\xi \pm i \bar{r}) S_{ \pm}(\xi ; \zeta)}
$$

At this point the factorization is almost complete. It remains to remove the pole in $G_{+}(\xi ; \zeta)$ at $\xi=i c$. This is accomplished by observing that

$$
\frac{G_{+}(\xi ; \zeta)}{\xi-i c}=\left[\frac{G_{+}(\xi ; \zeta)-G_{+}(i c ; \zeta)}{\zeta-i c}\right]_{+}+\left[\frac{G_{+}(i c ; \zeta)}{\zeta-i c}\right]_{-} .
$$

Now the factorization is complete and relationship (4.8) may be rewritten as

$$
\begin{aligned}
-\frac{\mu \kappa}{b^{2}} \frac{U_{-}(\xi ; \zeta)}{G_{-}(\xi ; \zeta)}-\frac{i P}{2 \pi v} \frac{G_{+}(i c ; \zeta)}{\xi-i c} & \\
& =\frac{i P}{2 \pi v} \frac{\left[G_{+}(\xi ; \zeta)-G_{+}(i c ; \zeta)\right]}{\xi-i c}+G_{+}(\xi ; \zeta) \Sigma_{+}(\xi ; \zeta) .
\end{aligned}
$$

The function on the left-hand side of $(4.11)$ is analytic in the half plane $\operatorname{Im}(\xi)<0$. The function on the right-hand side is analytic in the half plane $\operatorname{Im}(\xi)>-i \sqrt{\zeta^{2}+a^{2}}$. Since (4.11) holds in the strip where the domains of analyticity of the two functions overlap, the functions are each analytic continuations of the other. Together they define an entire function $E(\xi)([6]$, p. 37). The function $E(\xi)$ is to be determined from the behavior of the functions $U_{-}, \Sigma_{+}$, and $G_{ \pm}$as $|\xi| \uparrow \infty$. 
For the class of fracture mechanics problems described in Sec. 2, the normal stress $\sigma_{+}$ on the plane $y=0$ is expected to have the asymptotic behavior

$$
\sigma_{+}(x, z, t) \sim k_{I}(t, z) / \sqrt{2 \pi x} \quad \text { as } x \downarrow 0^{+}
$$

where $k_{I}(t, z)$ is the dynamic stress intensity factor history at any point along the crack front. This history will be determined explicitly in the next section. In light of (4.12), $\hat{\sigma}_{+}(x, z, s)$ is also expected to be square root singular as $x \downarrow 0^{+}$for any $z$. This result can be used in conjunction with the Abelian theorem regarding asymptotic behavior of Fourier transforms [6] to determine the asymptotic properties of $\Sigma_{+}(\xi ; \zeta)$, namely,

$$
\lim _{\xi \uparrow \infty} \sqrt{2 \xi / s} e^{-i \pi / 4} \Sigma_{+}(\xi ; \zeta)=\lim _{x \downarrow 0+} \sqrt{x} \hat{\sigma}_{+}(x, z, s) .
$$

Since the right-hand side of (4.13) is equal, by (4.12), to a function parametric in $s$ and $\zeta, \Sigma_{+}(\xi ; \zeta)=O\left(\xi^{-1 / 2}\right)$ as $\xi \uparrow \infty$. Furthermore, $u_{-}$is expected to vanish as $x \uparrow 0^{-}$and $\left|G_{ \pm}(\xi ; \zeta)\right|=O\left(|\xi|^{-1 / 2}\right)$ as $|\xi| \uparrow \infty$. Therefore, both sides of (4.11) vanish as $|\xi| \uparrow \infty$. That is, $E(\xi) \rightarrow 0$ as $|\xi| \uparrow \infty$. From the preceding discussion it follows that $E(\xi)$ is a bounded function in the entire $\xi$-plane. According to Liouville's theorem a bounded entire function is a constant [4], and therefore $E(\xi)$ must be identically zero, i.e., $E(\xi) \equiv 0$. Equation (4.11) can now be solved for $\Sigma_{+}$and $U_{-}$, which are found to be

$$
\begin{aligned}
U_{-}(\xi ; \zeta) & =\frac{P}{2 \pi i v} \frac{b^{2}}{\mu \kappa} \frac{G_{+}(i c ; \zeta) G_{-}(\xi ; \zeta)}{\xi-i c} \\
\Sigma_{+}(\xi ; \zeta) & =-\frac{P}{2 \pi i v} \frac{1}{(\xi-i c)}\left[\frac{G_{+}(i c ; \zeta)}{G_{+}(\xi ; \zeta)}-1\right] .
\end{aligned}
$$

This completes the formal solution of the problem. In theory, the exact solution to the problem is found by taking the triple inverse transforms of (4.14).

5. Inversion. Unfortunately, an exact inversion of $\Sigma_{+}$and $U_{-}$as given by (4.14) is not evident. This section is concerned with the determination of the stress intensity factor history, which is given by the asymptotic inversion of $\Sigma_{+}$with respect to $\xi$. As mentioned in Sec. 4, the asymptotic behavior of $\Sigma_{+}(\xi ; \zeta)$ as $|\xi| \uparrow \infty$ is intimately related to the behavior of the double transform $\hat{\Sigma}_{+}(x, \zeta)$ of the normal stress $\sigma_{+}(x, z, t)$ as $x \downarrow 0^{+}$. It follows from (4.12) that the Laplace transform of the dynamic stress intensity factor history $k_{I}(t, z)$ is simply

$$
\hat{k}_{I}(s, z)=\lim _{x \downarrow 0+} \sqrt{2 \pi x} \hat{\sigma}_{+}(x, z, s) .
$$

Thus, the double transform of $k_{I}(t, z)$ is found from (4.13) and (4.14) to be

$$
\hat{K}_{I}(s, \zeta)=\frac{P}{\pi v} \sqrt{\pi / s} \frac{\sqrt{c+\left(\zeta^{2}+a^{2}\right)^{1 / 2}}}{\left[c+\left(\zeta^{2}+r^{2}\right)^{1 / 2}\right] S_{+}(i c, \zeta)} .
$$

By construction, the function $\hat{K}_{I}(s, \zeta)$ is analytic on the strip $-i a<\zeta<i a$. Since the Wiener-Hopf factorization has been completed and the limiting process that resulted in $\hat{K}_{I}(s, \zeta)$ has suppressed all dependence on the variable $\xi$, there is no longer any need to restrict $\zeta$ to this strip. For the purposes of inverting this function it will be advantageous 
to extend the definition of the function to the entire $\zeta$-plane. It is this extended function which is referred to as the Fourier transform of $\hat{k}_{I}(s, z)$. This function can be made single-valued by cutting the $\zeta$-plane along $a<|\operatorname{Im}(\zeta)|<\infty, \operatorname{Re}(\zeta)=0$ and along $r<$ $|\operatorname{Im}(\zeta)|<\infty, \operatorname{Re}(\zeta)=0$. This ensures that the square roots have positive real parts. The function $S_{+}(i c, \zeta)$ is analytic over the entire $\zeta$-plane by virtue of a theorem pertaining to analytic functions defined by integrals ([4], p. 92; [6], pp. 11-12).

The inverse Fourier transform of (5.2) is

$$
\hat{k}_{I}(s, z)=\frac{s}{\sqrt{2 \pi}} \int_{-\infty+i \zeta_{0}}^{\infty+i \zeta_{0}} \hat{K}_{I}(s, \zeta) e^{-i s \zeta z} d \zeta,
$$

where $\zeta_{0}$ is a real number between $-a$ and $a$. It is hoped that the final Laplace inversion of $\hat{k}_{I}(s, z)$ can be done by means of the convolution formula. Therefore, it will be advantageous to cast (5.3) in the form of a one-sided Laplace transform so that the inversion can be performed by inspection. The required transformation can be achieved by suitably deforming the Fourier inversion path into a branch line integral; this is a trivial case of the Cagniard-deHoop technique. Consider the case of $z>0$. For (5.3) to be a convergent integral when $z>0$, it is necessary that the inversion contour lie in the lower half plane $\operatorname{Im}(\zeta)<0$. Since the finite $\zeta$-plane does not contain any singularities aside from the branch points at $\zeta= \pm i a$ and $\zeta= \pm i r$, the original inversion path can be deformed into a new contour as depicted in Fig. 5. This new contour consists of two quarter circles $\Gamma_{1}$ and $\Gamma_{2}$ in the lower half plane, and a branch line path running upward along the left-hand side of the branch cut from $-i \infty$ up to the branch point at $\zeta=-i a$, around the

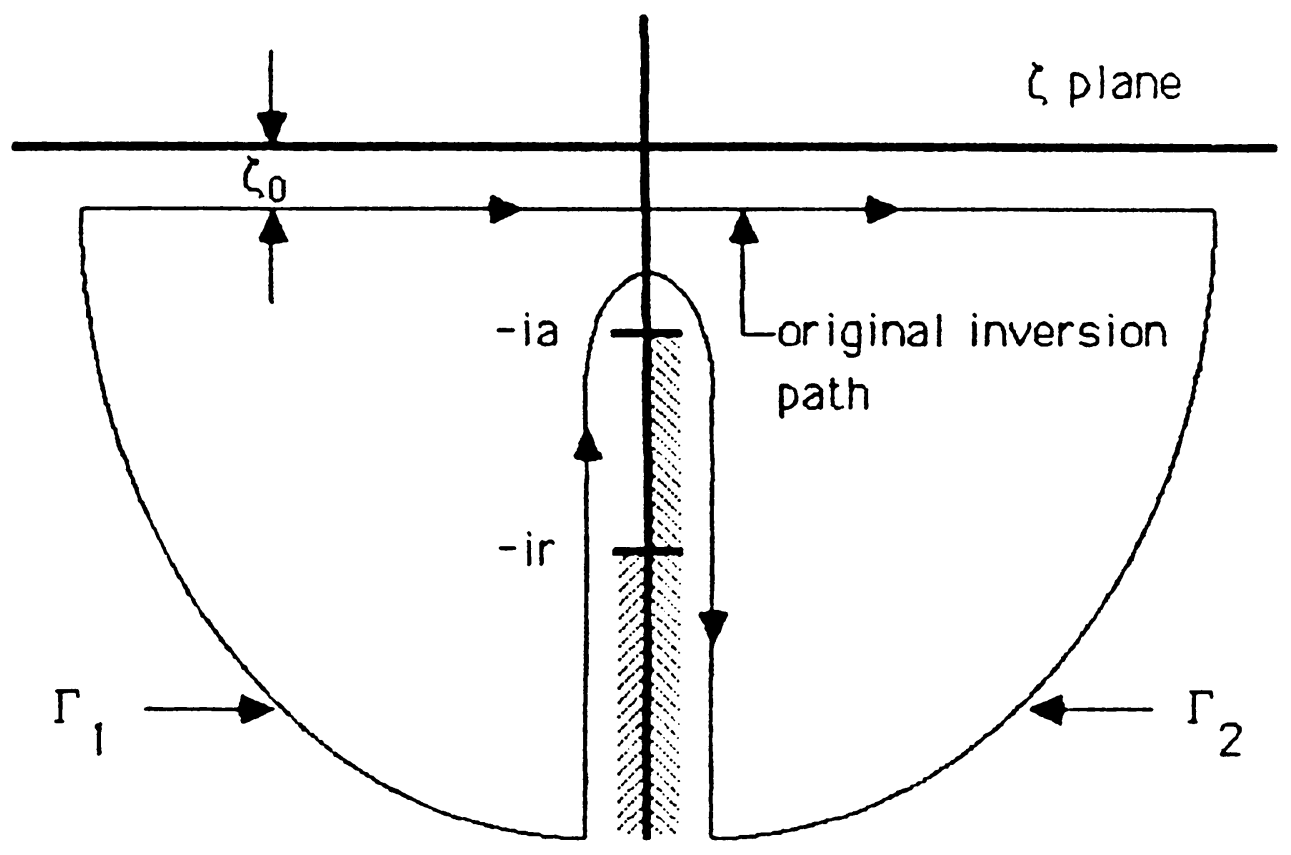

FIG. 5. The complex $\zeta$ plane, showing the singularities of $\hat{K}_{l}(s, \zeta)$ and the integration path for the evaluation of (5.3). 
branch point, and finally running down the right-hand side of the branch toward $-i \infty$. That the original integral in (5.3) is equivalent to the integral of the same argument along the new path follows from Cauchy's integral theorem [4]. The contribution of the integral along the arcs at infinity $\Gamma_{1}$ and $\Gamma_{2}$ as $|\zeta| \uparrow \infty$ vanishes by Jordan's lemma [4]. Thus (5.3) reduces to a branch line integral. Exploiting the fact that $\hat{K}_{I}(s,-\bar{\zeta})=\bar{K}(s, \zeta)$, where the bar denotes complex conjugation, the branch line integral can be expressed, after an elementary change of variables, as

$$
\hat{k}_{I}(s, z)=\frac{\sqrt{2} P}{\pi v} z^{-1} s^{-1 / 2} s \int_{a z}^{\infty} e^{-s \eta} \operatorname{Im}\{F(+0+i \eta / z)\} d \eta
$$

where

$$
F(\zeta)=\frac{\sqrt{c+\left(\zeta^{2}+a^{2}\right)^{1 / 2}}}{\left[c+\left(\zeta^{2}+r^{2}\right)^{1 / 2}\right] S_{+}(i c, \zeta)}
$$

In (5.4), $F(\zeta)$ is evaluated on the right-hand side of the branch cut. The $s$-multiplied Laplace transform in (5.4) can be expressed as the Laplace transform of a derivative, i.e.,

$$
\hat{k}_{I}(s, z)=\frac{\sqrt{2} P}{\pi v} z^{-1} s^{-1 / 2} \int_{a z}^{\infty} e^{-s \eta} \frac{\partial}{\partial \eta} \operatorname{Im}\{F(+0+i \eta / z)\} d \eta .
$$

There is no endpoint contribution from $\eta=a z$ in (5.5), because $F(+0+i a)$ is a real quantity. The inversion of (5.5) is now obvious because $\hat{k}_{I}(s, z)$ is seen to be the product of two transforms. Therefore $k_{I}(t, z)$ is the convolution integral of the inverses of the two transforms,

$$
k_{I}(t, z)=\frac{\sqrt{2} P}{\pi^{3 / 2} v} z^{-1} \int_{a}^{t / z} \frac{\partial}{\partial \zeta} \operatorname{Im}\{F(+0+i \zeta)\} \frac{d \zeta}{\sqrt{t-\zeta z}} H(t-a z)
$$

for $z>0$, and $k_{I}(t,-z)=k_{I}(t, z)$. The expression for $k_{I}$ in (5.6) apparently cannot be reduced further in terms of elementary functions. Some of the properties of the real integral (5.6) along with an interpretation of the results are discussed in the next section.

6. Conclusion. Even though the dynamic stress intensity factor history (5.6) cannot be evaluated in terms of elementary functions it can be evaluated numerically and some of its salient features can be obtained analytically. Figures 6,7,8, and 9 show the results of the numerical integration of the integral (5.6) for values of the ratio of the point load velocity to the Rayleigh wave speed of $0.8,0.6,0.4$, and 0.2 for a Poisson ratio of $\nu=0.3$ $\left(c_{1} / c_{r}=2.02\right)$. The time scale has been nondimensionalized so that $\tau=1$ corresponds to the arrival of the dilatational wave at the observation position $z$ along the crack front. The dynamic stress intensity factor has been normalized by premultiplying (5.6) by $P^{-1}(\pi z)^{3 / 2}$.

Following the sudden application of the point loads, a point $z$ along the crack edge is at rest until the arrival of the dilatational front. This front is compressive in nature and the crack faces respond to it by initially trying to close together. This is reflected by the stress intensity factor being negative initially. The initial jump in the dynamic stress intensity 


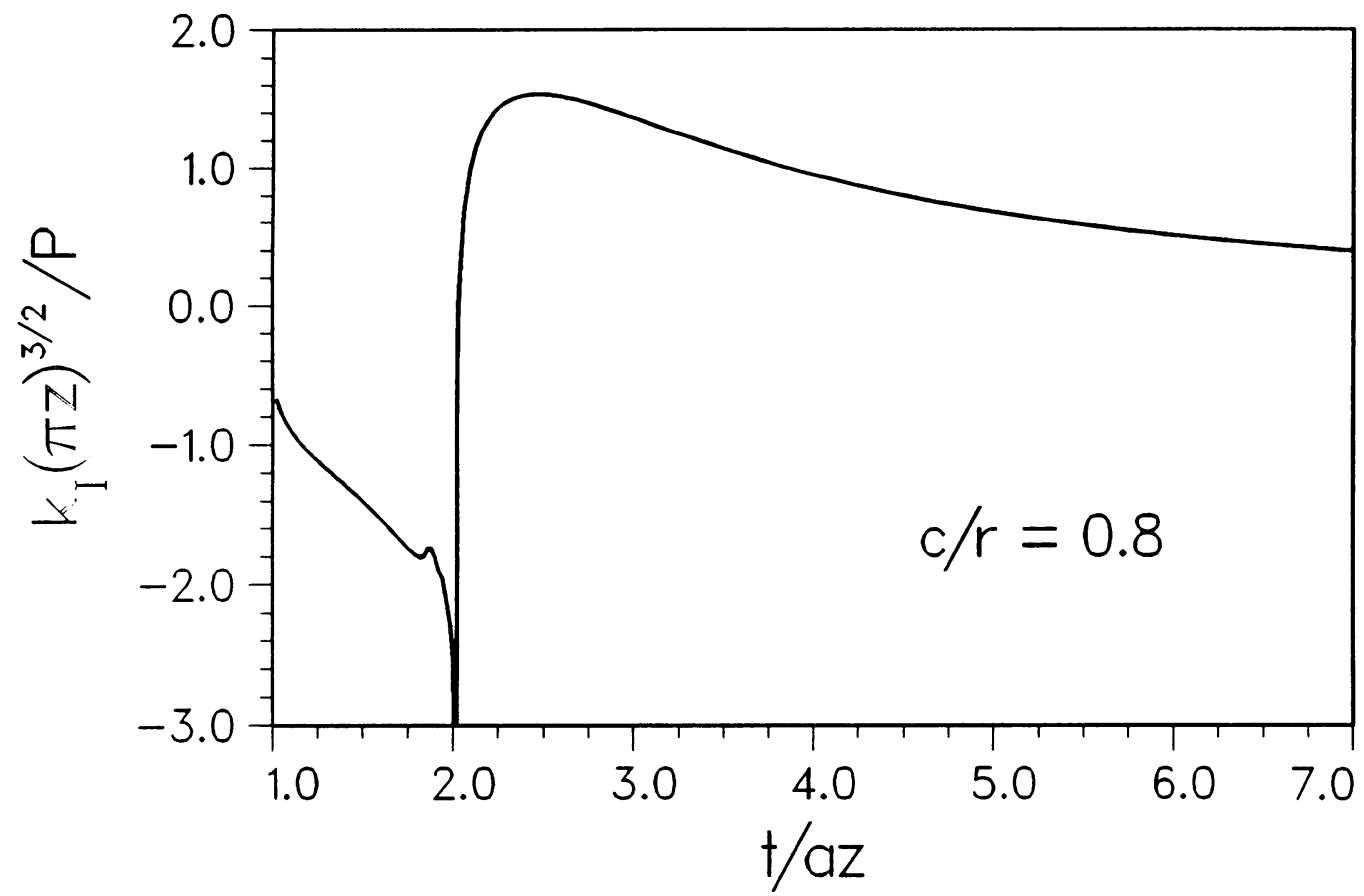

FIG. 6. The normalized stress intensity factor $k_{I}(t, z)(\pi z)^{3 / 2} / P$ versus $t / a z$ for the case of $c / r=0.8$.

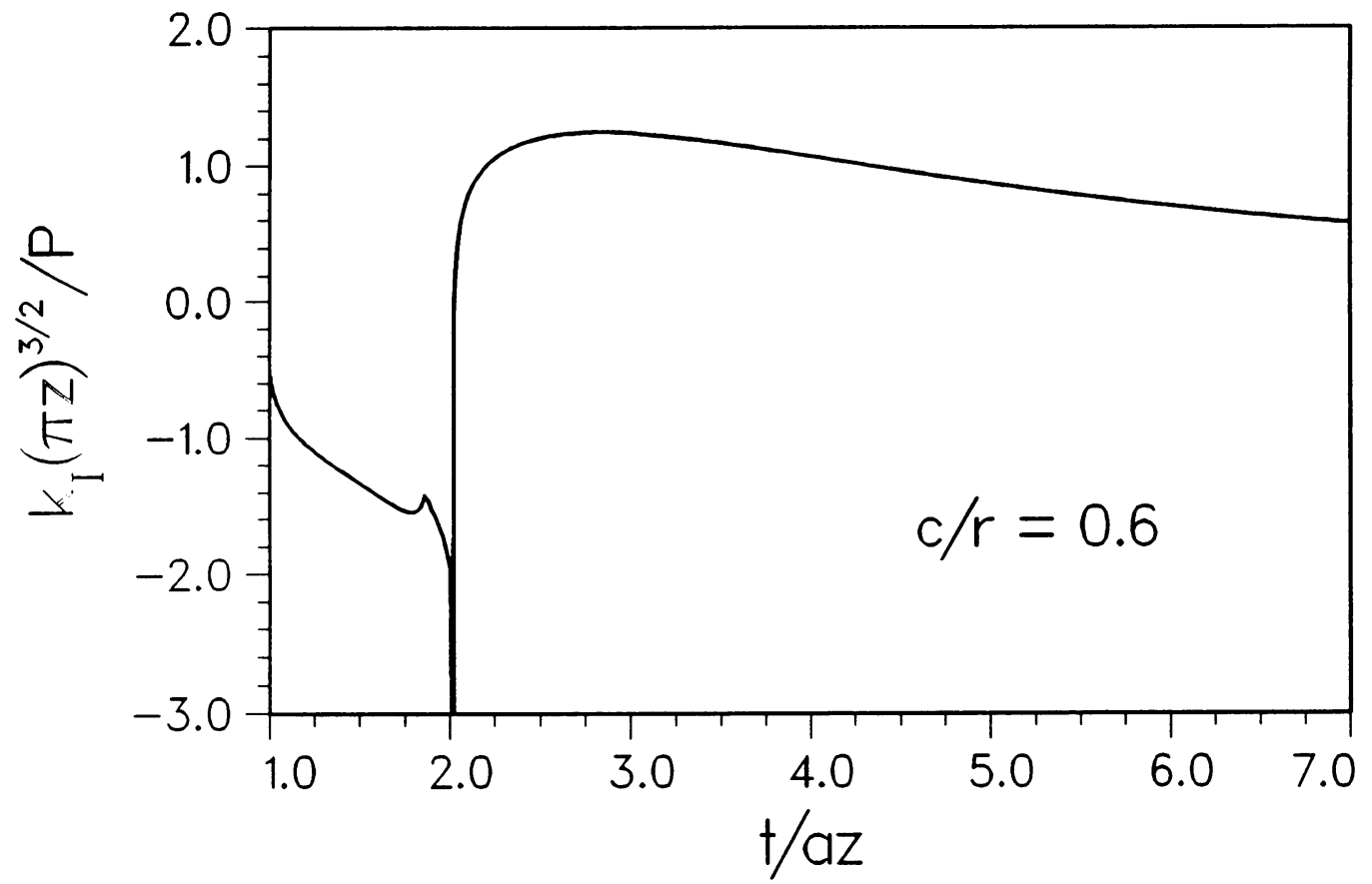

FIG. 7. The normalized stress intensity factor $k_{I}(t, z)(\pi z)^{3 / 2} / P$ versus $t / a z$ for the case of $c / r=0.6$. 


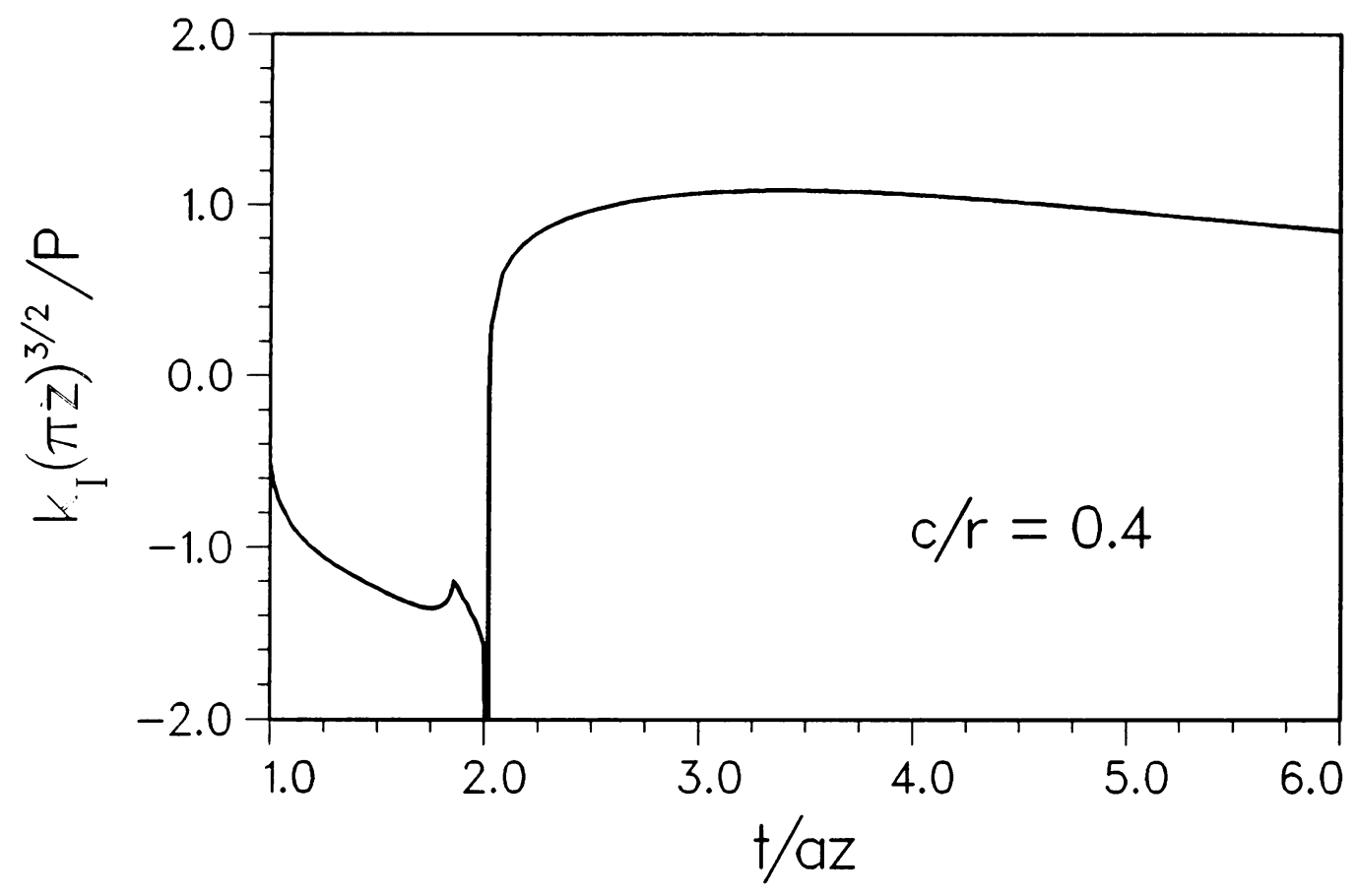

FIG. 8. The normalized stress intensity factor $k_{I}(t, z)(\pi z)^{3 / 2} / P$ versus $t / a z$ for the case of $c / r=0.4$.

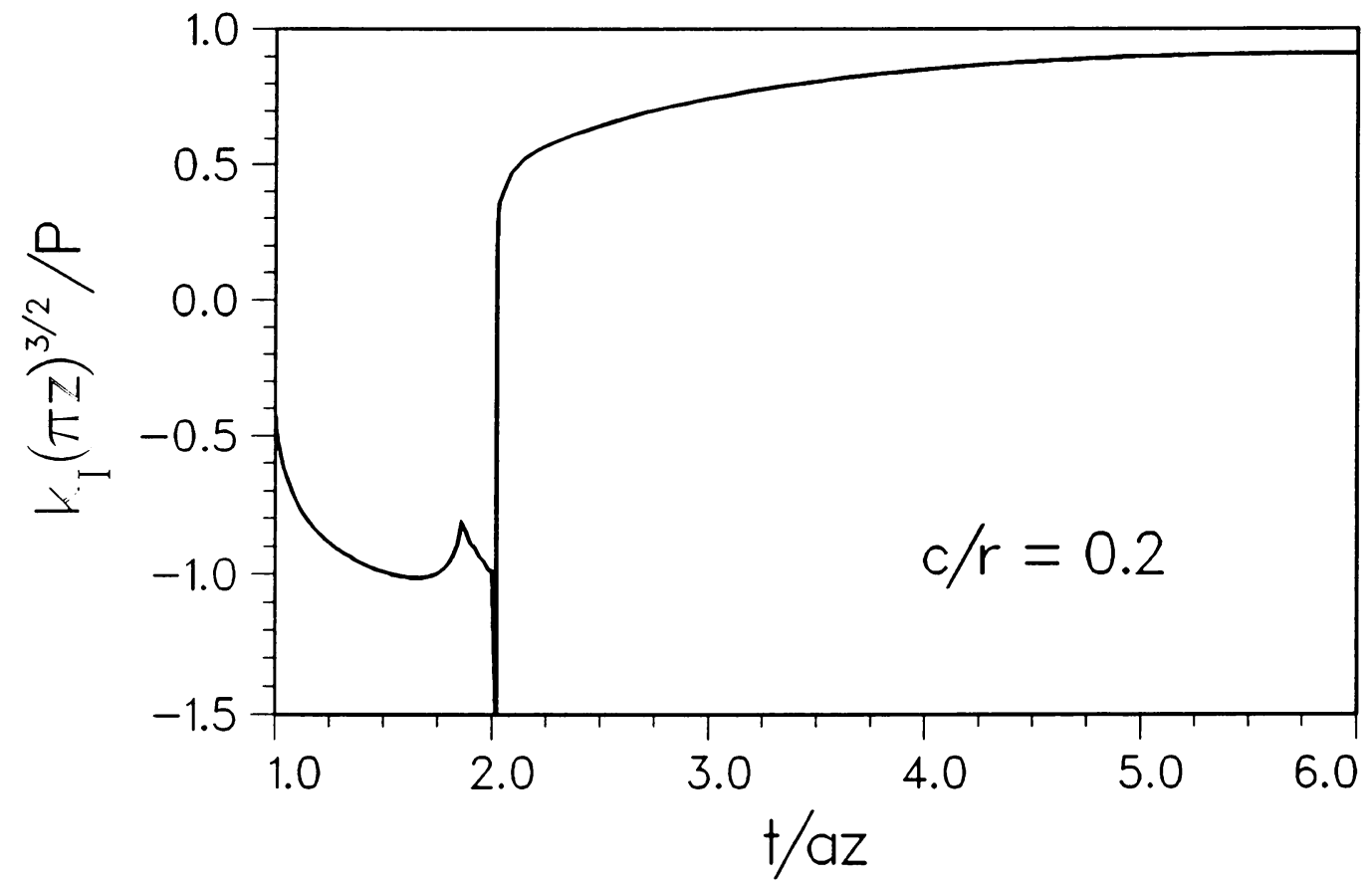

FIG. 9. The normalized stress intensity factor $k_{l}(t, z)(\pi z)^{3 / 2} / P$ versus $t / a z$ for the case of $c / r=0.2$. 
factor, which can be seen in the graphs, is a verifiable feature of the solution of this particular three-dimensional loading distribution. For the case of a pair of line loads acting perpendicular to the crack edge, i.e., the convolution of the traction distribution (4.1) for $v=0$, Freund [1] found that the dynamic stress intensity factor started from zero and gradually became negative. By taking the limit as $t / z \downarrow a^{+}$in (5.6) the jump is found to be

$$
\lim _{t / z \downarrow a^{+}} k_{I}(t, z)(\pi z)^{3 / 2} P^{-1}=-\frac{\pi}{4} \frac{\sqrt{4 a c}}{\left[c+\sqrt{r^{2}-a^{2}}\right] S_{+}\left(i c, 0^{+}+i a\right)}
$$

The dynamic stress intensity factor becomes increasingly negative until the arrival of the Rayleigh waves at $\tau=2.02$, when it becomes $\operatorname{logarithmically~singular.~Between~} \tau=1$ and $\tau=2.02, k_{I}(t, z)$ also exhibits a disturbance coinciding with the arrival of the shear front at $\tau=1.88$. This mild discontinuity in the slope of $k_{I}(t, z)$ is due to a change in the form of the function $S_{+}$as the branch point located at the shear wave slowness is crossed. After the passage of the Rayleigh waves the crack faces begin to open, and the stress intensity factor increases until it reaches a maximum and thereafter decays very gradually toward its limiting value $k_{I}(\infty, z)=0$.

This completes the analysis of the three-dimensional stress intensity factor history for the case of a pair of moving point loads on the faces of a crack. The solution (5.6) to the problem described by the traction distribution (4.1) is the fundamental solution to the class of problems involving traction distributions moving perpendicular to the crack edge. In this paper the range of applicability of the procedure introduced by Freund [1] has been successfully extended to this class of problems. Other situations, such as moving loads along a direction inclined to the crack edge, can be examined following the same methodology used in this paper.

Acknowledgment. I would like to extend my gratitude to Professor L. B. Freund for suggesting the problem treated in this paper. The research was possible thanks to the National Science Foundation, Solid Mechanics Program Grant No. MSM-85-13096, and the Office of Naval Research, contract N00014-85-k-0597, which provided access to the NRL Central Computer Facility.

\section{REFERENCES}

[1] L. B. Freund, The stress intensity factor history due to three dimensional transient loading of the faces of a crack, J. Mech. Phys. Solids 35, 61-72 (1987)

[2] D. D. Ang, Elastic waves generated by a force moving along a crack, J. Math. Phys. 38, 246-256 (1960)

[3] D. C. Gakenheimer and J. Miklowitz, Transient excitation of an elastic half space by a point load traveling on the surface, J. Appl. Mech. 3, 505-515 (1969)

[4] E. T. Whittaker and G. N. Watson, A course of modern analysis, Cambridge Univ. Press, 1927

[5] J. D. Achenbach, Wave propagation in elastic solids, North-Holland, Amsterdam, 1973

[6] B. Noble, Methods based on the Wiener-Hopf technique, Pergamon, Oxford, 1958 